\title{
POMPONAZZI Y LA ETERNIDAD DEL MUNDO. ENTRE EL PROBLEMA NEUTRO Y EL SABER DIALÉCTICO
}

\section{POMPONAZZI AND THE ETERNITY OF THE WORLD. BETWEEN THE NEUTRAL PROBLEM AND DIALECTICAL WISDOM}

\author{
JuAn MANuel ForTe \\ Universidad Complutense Madrid
}

Resumen: En el último capítulo del De immortalitate animae Pomponazzi afirma que el asunto de la inmortalidad del alma, como el de la eternidad del mundo, es un problema neutro. En este trabajo me gustaría mostrar que en realidad Pomponazzi no ha tratado la aeternitas mundi como un problema, sino más bien como una proposición verosímil en el sentido aristotélico. Por otro lado, se abordan algunos análisis que interpretan el pensamiento del filósofo a partir, entre otras, de estas cuestiones

Palabras clave: Pomponazzi, eternidad de mundo, naturalismo determinista, religión.

AвSTRACT: In the last chapter of De immortalitate animae, Pomponazzi claims that the question of immortality, just like the question of the eternity of the world, is a neutral problem. In this paper I claim that Pomponazzi has usually considered the aeternitas mundi as a probable proposition in the Aristotelian sense, rather than as a problem. Furthermore, I evaluate some analyses that use the former issues (among others) to interpret Pomponazzi's thought

Keywords: Pomponazzi, eternity of the world, deterministic naturalism, religion.

Pomponazzi no aborda la cuestión de la eternidad del mundo ${ }^{1}$ en su obra de un modo específico ni sistemático, aunque la plantea en numerosos textos

${ }^{1}$ La eternidad del mundo había sido una cuestión ampliamente debatida desde la incorporación de Aristóteles a la filosofía latina en el siglo XII. Sobre el desarrollo de la discusión hasta el 
y en relación con cuestiones diversas. Aparte de sus obras editadas, algunos de los textos donde más extensamente trata la cuestión, aunque siempre de forma secundaria, los hallamos en sus lecciones inéditas del periodo de su enseñanza en Padua (1488-96; 1499-1509), en su glosa del proemio de Averroes al III libro de la Física de Aristóteles (1514) y en sus comentarios al De coelo y en algunas lecciones inéditas de su enseñanza en Bolonia (1518).

En el caso de las lecciones de Padua, el problema es planteado fundamentalmente en la $4^{\mathrm{a}}$ cuestión del Super libello de substantia orbis expositio et quaestiones quattuor [1507]. Lo que se pregunta esta cuarta cuestión es «si Dios es la causa eficiente de todas las cosas o si sólo es la causa final» ${ }^{2}$. A partir del comentario de Averroes al libro XII de Metafísica, Pomponazzi postula que las sustancias separadas (substantiae abstractae) son causa final y formal del mundo, pero resulta problemático que sean causa eficiente. Frente a Avicena, que habría defendido que Dios es causa eficiente del mundo, Averroes sostuvo lo contrario mediante una reducción al absurdo: «si el mundo depende efectivamente de Dios, éste se hizo por medio de otra cosa o desde la nada»: si se hizo por medio de otra cosa, resulta que o bien no todo ente depende efectivamente de Dios, o que hay un proceso causal material infinito, lo que es imposible; en segundo lugar, que se hiciera desde la nada, es algo en patente contradicción con el pensamiento de Aristóteles, ergo... ${ }^{3}$ Ahora bien, continúa Pomponazzi, parece que de alguna manera Dios es causa eficiente del mundo, puesto que como señala el propio Averroes (en el comentario a los libros X y XII de Metafísica), el primer agente, el primer fin y la primera forma parecen poder identificarse, con lo cual Dios no sería sólo fin, sino forma y agente. Además, si Dios mueve al mundo en tanto que causa final, parece que es también de alguna forma causa eficiente de que el mundo se mueva. Por otro lado, continúa Pomponazzi, los comentaristas modernos asumen que si Dios hubiera creado efectivamente el mundo desde la

Renacimiento puede verse la antología comentada a cargo de C. Michon, Thomas d'Aquin et la controverse sur "L 'éternité du monde». Traités sur "L' Éternité du monde» de Bonaventure, Thomas d'Aquin, Peckham, Boèce de Dacie, Henri de Gand et Guillaume d'Ockham, París, Flammarion, 2004. Esta obra ofrece una amplia bibliografía. Véase asimismo el estudio general de R. C. Dales, Medieval Discussions on the Eternity of the World, Nueva York, Brill, 1990.

${ }^{2}$ La cuestión está orientada a refutar la concepción de Dios que piensa su relación con el mundo a través exclusivamente de la causalidad final (cfr. P. Pomponazzi, Corsi inediti del insegnamento padovano, q. IV, 113r-v, vol. I, p. 303). Si no se indica otra cosa, las traducciones de los textos latinos son del autor.

3 Ibid., vol. I, q. IV, 112, vol. I, p. 299. 
eternidad también podría volver a producirlo, lo que nuevamente sería absurdo y contrario a Aristóteles y al libro VIII de la Física. En efecto,

los vanos filósofos [fatui philosophi] opinaron que Dios actúa por necesidad de su naturaleza y, por tanto, puesta la causa adecuada de un efecto, debía seguirse un efecto necesario. Dios ha sido causa adecuada del mundo y, en tanto que actúa necesariamente, puesto Dios, se sigue afirmar un mundo desde la eternidad. Según los filósofos, no puede Dios producir innovadamente [de novo] el mundo, ya que, según ellos, esta afirmación es inconsistente, puesto que entonces lo nuevo provendría de un agente eterno e invariable, lo que según ellos no procede ${ }^{4}$.

El texto parte de la neta identificación entre la opinión de los «vanos filósofos» con la idea de un Dios que actúa por la necesidad de su naturaleza (no podemos descartar que ya en este tiempo el mantuano se considerara a sí mismo un «vano filósofo»). Por otro lado, la solución al problema, según Pomponazzi, debe partir de la distinción entre causa eficiente transmutativa (que presupone el sujeto transmutado) y una causa eficiente por analogía, que no presupone materia ni sujeto transmutable. Esto permite atribuir a Dios un tipo de causalidad eficiente (transmutativa) que no es incompatible con su carácter de eterno productor y conservador del mundo, aunque evidentemente se excluya la creación. Con todo, como el propio Pomponazzi recuerda en el último pasaje de la cuestión, Averroes no aceptó en ningún momento que la relación de Dios con el mundo fuera la de una simple creación tal y como la presentaban las tres religiones, ni que la creación pudiera haber surgido de la nada o innovadamente. Por su lado, Pomponazzi, siguiendo aquí la supuesta posición de Santo Tomás y Escoto, parece dejar abierta la puerta para una eterna emanación, producción y conservación por parte de Dios.

El anterior es uno de los textos más extensos en donde Pomponazzi aborda la eternidad del mundo, aunque siempre, como vemos, tangencialmente. Existen sin embargo otras muchas alusiones a la cuestión. Ya en sus lecciones de física anteriores, como en sus comentarios a la naturaleza del alma (entre los ańos 1499-1504), encontramos alusiones tanto a la concepción materialista y astrológicamente determinada del alma humana, como a la eternidad de la especie 5 .

${ }^{4}$ Ibid., vol. I, q. IV, p. 303.

5 P. Pomponazzi, Quaestiones Physicae et Animasticae Decem, q. III, 193r, en Corsi inediti dell'insegnamento padovano, vol. II, p. 82. 
Mucho más elocuente, pero no menos cautelosa, es su lección del Proemio de Averroes al libro III de la Física aristotélica. Aquí Pomponazzi expone la posición de Averroes sobre las relaciones entre religión y filosofía. Uno de los obstáculos para el desarrollo de la filosofía, de acuerdo con el Comentarista, procede de los errores antifilosóficos generados por la consuetudo (apuntalada a su vez por la teología espuria). En concreto, el error que supone negar un principio básico y central de la filosofía como es el de que «ex nibilo nibil fit ${ }^{6}$ ». Se trataría de un principio que se prueba a partir de la definición misma de movimiento que se halla en la Física del Estagirita: si el movimiento es el acto de un ente en potencia, y el hacerse de algo es un tipo de movimiento (una transmutación), éste supone un ente en potencia. Por consiguiente, nada puede surgir de la nada (puro no ente que carece obviamente de toda potencialidad), de ahí que Averroes esgrima una definición de movimiento de la naturaleza que implica su eternidad ${ }^{7}$. Ahora bien, este tipo de verdades filosóficas ponen en duda los artículos de fe de las leges, razón por la cual el teólogo y el legislador en general suelen ser enemigos de la filosofía ${ }^{8}$. Por último, como nos informa Bruno Nardi citando a Pomponazzi, éste se cura en salud recordando a sus alumnos «que el Comentador dice muchas futilidades, y que para que un corazón sincero se salve la sola fe es suficiente ${ }^{9}$. $\mathrm{Al}$ respecto de la aeternitas mundi, Pomponazzi menciona también que Galeno recuerda el primer libro de los Tópicos para determinar que es un "problema neutro», y que aunque él (Galeno) creía que era eterno, estaba dispuesto a aceptar lo que pensara la "canalla» con tal de no ser lapidado ${ }^{10}$. Y en su comentario del De Caelo, Pomponazzi recuerda con humor las consecuencias positivas de aceptar el relato del Génesis y negar la eternidad del mundo: "pues si el mundo no fuera nuevo y no hubiera existido el paraíso terrestre y quelle altre belle cose ... los frailes no harían penitencia, ni comerían habas, ni se morirían de frío ${ }^{11}{ }$.

Otro ejemplo, algunos años después, lo hallamos en una de las lecciones inéditas de 1518 (Quaestio de genitis ex putri materia ${ }^{12}$ ), un comentario al texto

${ }^{6}$ P. Pomponazzi, In proemium Commentatoris, en Bruno Nardi, Studi su Pietro Pomponazzi, Florencia, Le Monnier, 1965, p. 144.

7 Ibid. p. 144.

8 Ibid., pp. 147-48.

9 Ibid., p. 136.

10 Ibid., p. 136.

11 Ibid., p. 138.

12 P. Pomponazzi, Quaestio de genitis ex putri materia (1518), en Vittoria Perrone, «La stagione delli frumenti. Due lezioni di Pomponazzi sulla generazione spontanea», Bruniana \& Campanelliana, 17/1, 2011, pp. 199-222. 
aristotélico «Sobre la corrupción y la generación». En este caso Pomponazzi analiza tres posiciones en torno a la generación de los seres vivos y más particularmente del hombre. En primer lugar, la posición de Averroes (y de Aristóteles) de acuerdo con la cual parece imposible la generación «espontánea» a partir de la materia inerte (ex putri materia), siendo necesaria la sucesión por propagación (ex propagine $\left.{ }^{13}\right)$. Por otro lado, Avicena en su De diluviis habría sostenido la posición opuesta: todas las especies pueden generarse ex putri materia y algunas (sobre los pequeños organismos) sólo de esta manera ${ }^{14}$. Podría ser que en el curso de los tiempos los hombres hubieran perecido debido a los cataclismos de agua o fuego o por su propia abstinencia copulativa. Si esto es así, la especie humana pereció en el pasado, volviendo a generarse a partir de la materia putrefacta. Ni puede pensarse de otro modo si se combina esta idea de los cataclismos periódicos por agua y por fuego con la idea de la eternidad del mundo ${ }^{15}$.

Aunque la posición de Avicena no pueda demostrarse e incluso a Pomponazzi le parezca más sensata una tercera alternativa ${ }^{16}$ (algunos seres se generan ex propagatione, otros ex putredine, otros aún, utroque modo) el mantuano la defiende de las críticas de Averroes (basadas a su vez en Aristóteles) que pretenden hacerla pasar por absurda. En este sentido, tanto desde el punto de vista de su racionalidad, como de lo que se deduce de la experiencia, la hipótesis de Avicena no resulta improbable por lo que respecta a los pequeños organismos, ni imposible (aunque si menos probable) por lo que respecta a la generación de grandes animales.

Trasladándonos ahora a sus obras centrales, en el De immortalitate animae, Pomponazzi indica y acepta que según la filosofía de Aristóteles el mundo y el hombre son eternos ${ }^{17}$. Y asimismo, en el Epílogo del De fato, se vuelve a aludir a la cuestión cuando se reitera la ausencia de inconvenientes que se derivarían de negar la libertad del albedrío. Así, nos dice Pomponazzi, en el primer miembro de una reducción al absurdo, si no existiera el libre albedrío y las almas fueran mortales (como creían los estoicos y el propio Pomponazzi ya postulaba en el De immortalitate animae) el universo estaría perfectamente ordenado por el equilibrio de buenos y malos, de lobos y ovejas: «este orden ha sido por los siglos infinitos, y lo será por infinitos, porque

13 P. Pomponazzi, Quaestio de genitis ex putri materia, pp. 204-206.

${ }_{14}$ Ibid., pp. 206-7.

15 Ibid., pp. 207.

16 Ibid., pp. 216-7.

17 P. Pomponazzi, Tratado sobre la inmortalidad del alma, ed. de J. M. García Valverde, Madrid, Tecnos, 2010, VIII, p. 57. 
siempre tiene una causa necesaria y per se; por lo que [el orden del mundo] no está en nuestra potestad, sino en poder del destino ${ }^{18}$ ». En segundo lugar, si no hubiera libre albedrío y las almas fueran inmortales, continúa Pomponazzi, sería necesario introducir la idea de la reencarnación de las almas en los cuerpos y el infinito reparto de papeles, con lo que la justicia cósmica estaría salda ${ }^{19}$ siempre que se supusiera el giro infinito de los siglos en el marco de la aeternitas mundi.

Por último, en el De incantationibus, habiendo argumentado a favor de la naturaleza corruptible y material de las religiones, sujetas a la determinación astral, Pomponazzi asume esta determinación como una hipótesis probable en la medida en que permite explicar en términos naturales y clave peripatética todos los fenómenos excepcionales asociados a ellas: los milagros, los portentos, los oráculos, la profecía y la eficacia de los ruegos a Dios. Frente a los que piensan que todo esto procede de los ángeles o los demonios, «parece más verosímil, que esto se produzca a partir de la disposición de los cuerpos celestes, en virtud principalmente del movimiento que Dios y las inteligencias imprimen en tales cuerpos celestes ${ }^{20}$ ». A lo que el filósofo añade que todo ello, «incluso no siendo cierto", se corresponde con el testimonio de las historias y con los escritos de los filósofos: "puesto que según los filósofos (en especial según Platón y Aristóteles) el mundo es eterno ${ }^{21} »$, de manera que este género de cosas han sido infinitas y seguirán infinitamente siendo en el futuro ${ }^{22}$ (en cuanto a la especie, no en cuanto individuos). Las ceremonias del presente ya fueron en número infinito en el pasado y se darán en número infinito en el futuro. «Así que si esta mudanza [de cultos, ceremonias y los prodigios asociados] es incesante y eterna, tiene que tener una causa eterna en sí misma, y esta causa no puede residir en nada más que en los cuerpos celestes, Dios y las Inteligencias ${ }^{23} »$. De igual modo, la máquina celeste ha determinado las vicisitudes de las monarquías, los reinos, las ciudades y, en fin, de todas las «magnas mutaciones ${ }^{24}$ » La cuestión fundamental, no debe dejar de referirse, es justamente que los prodigios, los milagros no acaecen «contra la naturaleza, ni son ajenos al orden de los cuerpos celestes ${ }^{25}$ ». Los

18 P. Pomponazzi, Libri Quinque de Fato, de Libero Arbitrio et de Praedestinatione, ed. de R. Lemay, Lugano, 1957, p. 451.

19 Ibid., p. 452.

20 P. Pomponazzi, De incantationibus, ed. de V. Perroni, Florencia, Olschki, 2011, XII, p. 152.

${ }^{21}$ Ibid., XII, p. 152.

22 Ibid., XII, p. 153.

${ }^{23}$ Ibid., XII, p. 153.

24 Ibid., XII, p. 153.

25 Ibid., XII, p. 154. 
milagros suceden de acuerdo con el orden natural, aunque sean raros y escasos y requieran vastos periodos de tiempo. En otras palabras, el milagro pierde aquí ya toda connotación «sobrenatural», para convertirse en un efecto natural, aunque extremadamente raro.

De todo lo anterior, se sigue, en primer lugar, que Pomponazzi parece asumir la eternidad del mundo como una premisa básica in via peripatética, de acuerdo también con la razón natural. En la medida en que Dios está determinado por su propia naturaleza que es puro acto, eterno e inmutable, y en la medida en que Dios es causa adecuada (final, formal y eficiente en cierto sentido) del mundo, puesta la causa, está puesto el mundo, y resulta en cambio absurdo pensar en un mundo nacido ex novo, de novo y mucho menos ex nihilo. Lo mismo podría fundamentarse, como hemos visto, a partir del concepto aristotélico de movimiento.

En segundo lugar, según Pomponazzi, se trata de una cuestión hipotética, conjetural, de la que no cabe demostración apodíctica ni certeza. Es, en cierto sentido «un problema». Eso dice expresamente el mantuano al comparar la cuestión de la inmortalidad del alma con la de la eternidad del mundo: «Por lo tanto, estando así las cosas, a menos que se dé un parecer más acertado, yo creo que en este asunto se ha de decir que la cuestión de la inmortalidad del alma es un problema neutro, exactamente como el de la eternidad del mundo. Y es que, a mi parecer, ninguna razón natural puede ser aducida que demuestre que el alma es inmortal, y tanto menos que prueben que el alma sea mortal, tal y como declaran muchos doctores que la consideran inmortal ${ }^{26}{ }_{\text {». }}$.

Equiparación neta entre eternidad del mundo e inmortalidad del alma: dos cuestiones neutrales, dice Pomponazzi, equidistantes, por consiguiente, respecto a la certeza apodíctica en una dirección u otra de sus polos dilemáticos. Esta afirmación, no obstante, produce una cierta extrañeza, porque si Pomponazzi ha dedicado un tratado a mostrar que la inmortalidad del alma no admite demostración racional, y que su mortalidad es cuanto menos "posible» desde el punto de vista de la razón, y probable in via peripatetica, la aternitas mundi, por su lado, no parece estar en el mismo plano, o al menos en la obra del mantuano tiene un tratamiento muy diferente. En efecto, como hemos visto, la eternidad del mundo no ha sido abordada como una «cuestión» a dilucidar, sino más bien como una

26 P. Pomponazzi, Tratado sobre la inmortalidad del alma, XV, p. 162. He alterado ligeramente la traducción de Valverde (haciéndola más literal), aunque su versión respeta plenamente el sentido del texto. 
proposición ${ }^{27}$ que merece pocas explicaciones: «según los principios de Aristóteles el mundo es eterno y el hombre es eterno ${ }^{28}$ ". Proposición que Pomponazzi emplea, como hemos visto, como premisa de sus argumentaciones y reductiones ad absurdum sobre diversas materias. Poco importa aquí que Aristóteles en sus Tópicos no diga exactamente (como pretende Galeno y ahora Pomponazzi) que el problema de la eternidad del mundo sea neutro, sino más bien que es una cuestión difícil de decidir por su amplitud, y por el esfuerzo argumentativo que exige $^{29}$. Lo importante es que a pesar de la equiparación entre inmortalidad del alma y eternidad del mundo, está última no se le plantea a Pomponazzi como un problema, sino más bien como una premisa aristotélica.

Por lo demás, el final del Tractatus introduce una corrección que no sabemos bien si completa el cuadro que Pomponazzi nos ha presentado o lo trastoca enteramente. Al fin y al cabo, Dios, afirma el filósofo, ya antes de la venida de Cristo, ha desbaratado la neutralidad de la cuestión sobre la inmortalidad, dilucidando su solución: «se ha de aceptar pues, sin lugar a dudas que el alma es inmortal ${ }^{30}$ ». Los que avanzan por el camino de la razón, por el camino de los «saeculi sapientes», están condenados, como arengó Pablo, a la insensatez. Quien persiste en esta vía, fluctuará "incertus et vagus ${ }^{31}$ », como habría sido el caso del mismo Platón. En consecuencia, las argumentaciones que parecen probar que el alma es mortal son "falsae et apparentes» y lo mismo, puede fácilmente colegirse, podría entonces decirse de la eternidad del mundo.

Es bien sabido, y ha sido ampliamente debatido, que este tipo de palinodias está presente en todas las obras controvertidas de Pomponazzi y también, como hemos visto, en algunas de sus lecciones. Si se aceptan en su literalidad, implican que, sin la fe, el filósofo mundano está condenado a una «vana ambición», y a la incertidumbre generada por el estudio de realidades naturales que, por

${ }_{27}$ Para la diferencia entre proposición y problema, cfr. Aristóteles, Tópicos, trad. de M. Candel, Gredos, Madrid, 1982, I, 10, $104 \mathrm{a}$.

28 P. Pomponazzi, Tratado sobre la inmortalidad del alma, XV, p. 57.

29 «Son también problemas aquellas cuestiones de las que hay argumentaciones contrarias (...) y aquellas cuestiones acerca de las cuales, por ser muy amplias, no tenemos argumentos, juzgando que es difícil dar el porqué de ellas, v.g.: la de si el mundo es eterno o no». Aristóteles, Top., I, 11, $104 \mathrm{~b}$.

30 P. Pomponazzi, Tratado sobre la inmortalidad del alma, XV, p. 167.

31 Ibid., p. 167. Lo cierto es que (en otro capítulo) Pomponazzi defiende el valor de la verdad (la mortalidad del alma) frente a la tranquilidad: «el sabio elegiría estar en una extrema necesidad y en unas máximas tribulaciones antes que ser un tonto, indolente y vicioso, en la disposición que le es contraria» (Ibid., XIV, p. 133). 
definición, sólo admiten un conocimiento «oscuro y no firme ${ }^{32} »$. Si esto fuera la última palabra, la retractatio obligaría a la interpretación escéptica y fideísta del pensamiento de Pomponazzi. Fideísmo, en efecto, puesto que la inmortalidad del alma (así como, ya lo hemos dicho, otras tantas cuestiones semejantes) no es sino un articulus fidei, cuya certeza se obtiene sólo mediante la fe, y que no admite demostraciones racionales e impertinentes en relación a la cuestión. Escepticismo, también, porque todo el esfuerzo de la razón del que resultan verosimilitudes incompatibles con la fe y la dogmática católica es estéril y vacuo: la eternidad del mundo, la naturaleza necesaria de Dios, la ausencia del libre albedrío, la autosuficiencia de la moralidad sin trascendencia ni inmortalidad, la explicación naturalista de los milagros, el horóscopo de las religiones, etc. Cuestiones que, al cabo, sólo sirven para ejercitarse en un virtuosismo especulativo inane. Así, por ejemplo, asumiendo la literalidad del epílogo del Tratado sobre la inmortalidad del alma, García Valverde concluye que el supuesto descreimiento y proto-libertinismo de Pomponazzi es insostenible, primero, por razones inmanentes a su discurso, esto es, por su agnosticismo expreso; segundo, por razones extrínsecas de carácter histórico y social: «no nos parece comprensible el hecho de que, pese a la polvareda que, en efecto, produjo la publicación De immortalitate animae, su autor no fuera condenado ni tuviera que retractarse; es más, en las obras que escribió en su defensa no hace sino reafirmarse en sus anteriores pareceres ${ }^{33}{ }$. En realidad, esta es más o menos la línea de defensa que Pomponazzi hace expresamente de su caso en su Apologia. Primero, en cuanto al carácter ortodoxo de su posición: «yo estoy dispuesto a morir para afirmar la inmortalidad de las almas ${ }^{34}$ ». Segundo, porque jamás ha sido reprendido por los que velan por la ortodoxia ${ }^{35}$. Por último, porque nadie puede acusar a Pomponazzi de ser un mal cristiano:

Examínese mi vida, tanto en las palabras como en los actos. Nadie podrá nunca acusarme de herejía ni cuando enseño ni cuando discuto públicamente ni cuando converso en privado: nunca ha salido nada de mi boca que no fuera cristiano. Siempre, en la medida de mis posibilidades, he tratado de

32 Ibid., p. 166.

33 J. M. García Valverde, Estudio Preliminar, en P. Pomponazzi, Tratado sobre la inmortalidad del alma, ed. cit. p. XCIV. Antonino Poppi parece mantener una tesis parecida en un reciente artículo, describiendo a un Pomponazzi bifronte, afiliado al fideísmo teológico al tiempo que al reduccionismo materialista (A. Poppi, "Consenso e dissenso del Pomponazzi con il subtilissimus et religiosissimus Ioannes Scotus", en Pietro Pomponazzi. Tradizione e dissenso, ed. de M. Sgarbi, Florecia, Olschki, 2010, p. 39).

34 P. Pomponazzi, Apologia, ed. de V. Perrone, Florencia, Olschki, 2011, III, 1, 3, p. 248.

35 Ibid., III, 3, 2, p. 257. 
combatir las afirmaciones que resultan adversas a la religión cristiana: testigos a mi favor son Padua, Ferrara y Bolonia ${ }^{36}$.

Sin embargo, esta interpretación se presta a serias dudas. Para empezar, porque en relación con la coherencia interna de su discurso es precisamente esto lo que cada texto de Pomponazzi parece desafiar. En realidad, la coherencia interna de su pensamiento parece impecable tanto si la interpretamos desde el escepticismo cristiano, como si lo hacemos desde un racionalismo enmascarado. Y los mismos motivos de duda concitados por el De immortalitate animae se repiten en el De fato y el De incantationibus, que no por casualidad permanecieron inéditos por voluntad del autor. Por lo que respecta al argumento "externo", basado en la ausencia final de represalias, tampoco parece concluyente, porque en vez de señalar que Pomponazzi no fue procesado, también podríamos subrayar que Pomponazzi ya había sido acusado (seguramente por algún oyente de sus lecciones) en 1514 de herejía, precisamente por su exposición de Averroes en este punto de las relaciones entre filosofía y religión. Por lo demás, del proceso abierto en Venecia sí se derivaron consecuencias: como mínimo, el filósofo se vio obligado a escribir una Apologia y a poner en marcha todos los resortes a su alcance para protegerse. En este sentido, como él mismo indica en la Apologia, fue el apoyo expreso de Pietro Bembo (Secretario personal de León X) lo que determinó que la acusación de herética pravedad no prosperase ${ }^{37}$. Las sospechas continuaron, no obstante, como se infiere del hecho de que el Papa le exigiera una retractatio, y por el hecho de que no se atreviera a publicar en vida sus dos obras más polémicas (el De incantationibus y el De fato) ${ }^{38}$. Por otro lado, nadie puede ignorar que la persecución doctrinal no era precisamente la primera preocupación de León $\mathrm{X}^{39}$ (centrado más bien en cuestiones de índole financie-

${ }^{36}$ Ibid., III, 3, 7, p. 260.

37 Ibid., II, 1.

38 Sobre la actitud de Pomponazzi y de la Iglesia al respecto, véase el capítulo dedicado a esta cuestión en L. Bianchi, Pour una historie de la «doublé vérité», París, Vrin, 2008, pp. 117-134.

39 Para hacerse una idea de Leon X y su tesón doctrinal, bastaría con recordar el juicio de un contemporáneo suyo tan bien informado como Guicciardini. Recuerda éste su liberalidad con el dinero y los beneficios, «que envilecía la autoridad espiritual, desordenaba las formas de la corte... y le obligaba a buscar siempre dinero por vías extraordinarias». A esto se le ańadía, dice Guicciardini, una "profundísima simulación», y una gran afición a «a la música y a las burlas de los bufones», en cuyos menesteres se ocupaba «la mayor parte del tiempo». Se creyó al principio, continúa el historiador florentino, que se trataba de un "hombre castísimo", aunque con el tiempo se le descubrió «excesivamente entregado, y cada día con menos recato, a aquellos placeres que por decoro no pueden ser nombrados» (F. Guicciardini, Storia d'Italia, ed. de S. Seidel Mechi, Turín, Einaudi, 1971, XVI, 12, p. 1589). También Pastor (no precisamente sospechoso de anticlericalis- 
ra), y que Bembo (tan atareado en su vida amorosa, sus estudios ciceronianos y sus poemas petrarquescos) estaba lejos de ser precisamente un severo censor. Si se desea escuchar a un censor más escrupuloso, léanse las denuncias del Opusculum contra P. Pomponatium de Bartolomeo Spina (éste sí, un inquisidor de verdad); y si lo que se busca es una Roma que sí se tomó algo más en serio la censura doctrinal, pónganse los ojos en la Iglesia postridentina, esa que incluyó el $D e$ incantationibus en el Index librorum prohibitorum.

En cualquier caso, algo unánimemente aceptado es que no puede incluirse a Pomponazzi entre las filas del racionalismo católico tal y como lo encontramos en Santo Tomás o más radicalmente en Buenaventura de Fidanza o, por ejemplo, en el Nifo del Tractatus de immortalitate animae contra Pomponatium ${ }^{40}$. Esto es, una actitud orientada a señalar el carácter absurdo de los filosofemas que van contra los artículos fundamentales de la fe (así, San Buenaventura intenta demostrar que la idea de la eternidad del mundo es absurda e irracional), o a mostrar el carácter probable de los principios racionales que son concordes con la fe (Santo Tomás, en este mismo caso, argumenta que la eternidad del mundo es posible desde el punto de vista de la razón, pero también lo es su temporalidad $\left.{ }^{41}\right)$. Desde luego, Pomponazzi realiza su trabajo al margen de cualquier preocupación teológica o concordista, sordo por consiguiente ante las recomendaciones de la célebre bula Apostolicis regiminis de $1513^{42}$.

mo) recuerda los deleites «demasiado mundanos» de León X; su rodearse de «artistas, poetas, músicos, comediantes, bufones»; y sus desmedidos gastos en «juegos, teatros y cacerías» (L. Pastor, Historia de los papas, ed. de R. P. Ramón Ruiz, vol. VIII, Barcelona, Gusavo Gili, 1910, pp. 360-1). Y más expresivo aún, Falconi traza una colorida comparación con Lutero, que sintetiza la actitud mundana y despreocupada de la corte de León X (C. Falconi, Leone X: Giovanni de Medici, Milán, Rusconi, 1987, pp. 360-1).

${ }_{40}$ No obviamente porque llegue a conclusiones opuestas tanto en su análisis de la cuestión como en su interpretación de Aristóteles, sino por el espíritu que anima su producción teológica: confutar todos los errores de los filósofos, en especial aquéllos que fueron expresados «adversus christianam veritatem» (A. Nifo, L'immortalità dell'anima, ed. de J. M. García Valverde, Milán, Aragno, 2009, dedic., p. 4). Y en este mismo sentido se decanta su juicio de valor sobre el mérito de los filósofos cristianos: «al que de verdad cree en un artículo de fe no porque sea demostrable, sino porque nos fue transmitido por hombres santos, y luego lo prueba exponiéndolo por vía demostrativa, a ése juzgo digno de mérito» (ibid., cap. LXXXIV, p. 432).

41 Sobre las posiciones de Santo Tomás y Buenaventura resulta particularmente aclaratorio el magnífico artículo de F. J. Kovach, «The Question of the Eternity of the World in St. Bonaventure and St. Thomas", Southwestern Journal of Philosophy, 5(2), 1974, pp. 141-172.

42 De hecho hay algunos párrafos de la bula que parecen un traje a la medida del profesor de Mantua: «En nuestros días (con dolor lo admitimos) el sembrador de cizaña, el antiguo enemigo del género humano, ha osado diseminar y nutrir algunos perniciosísimos errores (siempre rechazados por los fieles) en el campo del Señor, en especial sobre de la naturaleza del alma racional, 
Un punto de partida diferente consiste en asumir que la verdadera posición de Pomponazzi es un asunto imposible de resolver sin servirnos de meras especulaciones sin fundamento. Quién más ha desarrollado esta línea interpretativa ha sido probablemente Vittoria Perrone en su introducción al De immortalitate, entre otros lugares. Por un lado, nos señala la estudiosa a propósito de este último capítulo del De immortalitate animae, el texto es formalmente irreprochable (ineccepibile) y objetivamente no contradictorio. Así las cosas, es imposible establecer «si lo que Pomponazzi escribe es lo que verdaderamente piensa ${ }^{43}$. En cualquier caso, para Perrone no hay ninguna incoherencia que permite una lectura del capítulo como «una sumisión insincera a la autoridad de la Iglesia ${ }^{44} »$. La postura del filósofo mantuano es en definitiva que la mortalidad del alma (y la eternidad del mundo) es desde el punto de vista filosófico probabilior respecto a la inmortalidad, pero puesto que no hay demostraciones racionales apodícticas en un sentido u otro, la cuestión se mantiene relativamente neutra ${ }^{45}$. Por otro lado, de acuerdo con Perrone, para Pomponazzi la razón y la fe son dos ámbitos absolutamente distintos, que nunca se encuentran en el terreno metodológico, porque la religión se mueve en el ámbito de la certeza procedente de la revelación (y que en este caso prueba la inmortalidad); la filosofía, por su lado, se mueve en el ámbito de probabilidad, que en este caso se orienta a afirmar, aunque no taxativamente, la mortalidad. Entre estos dos planos no hay comunicación posible, por lo que ni la fe debe sustentarse sobre el razonamiento filosófico (ni pretender limitarlo), ni la filosofía debe

por ejemplo, que es mortal o única en todos los hombres; y algunos que filosofan imprudentemente aseveran que esto es verdad al menos según la filosofía; así que deseando nosotros poner los oportunos remedios contra esta peste, con aprobación de este sagrado Concilio, condenamos y reprobamos a todos los que afirman que el alma intelectiva es mortal o única en todos los hombres, y a los que hacen de esto algo incierto(...) Y puesto que la verdad no puede en absoluto contradecir a la verdad, cualquier afirmación contraria a la verdad iluminada por la fe la definimos como absolutamente falsa, y puesto que no es lícito enseñar otra cosa, lo prohibimos taxativamente(...)». A continuación se conmina a los filósofos que enseñen cuestiones como la «mortalidad del alma» o la «eternidad del mundo» a poner todo su empeño en demostrar «la verdad de la religión cristiana». Sacrorum conciliorum nova et amplissima collectio, ed. de G. D. Mansi, París, H. Welter, 1901-1927, 53 vols., 1902, vol. 32, c. 843. Sobre el impacto de la Apostolicis regiminis y Pomponazzi, cfr. L. Bianchi, Pour una historie de la "double vérité», p. 119 y ss.

43 V. Perrone, «Introduzione», en P. Pomponazzi, Trattato sull'immortalità dell'anima, ed. de V. Perrone, Florencia, Olschki, 1999, p. LXXXVII.

${ }^{4}$ Ibid., p. LXXXVIII.

45 Ibid., p. LXXXIX. Creo que quien ha expresado este punto con más ingenio ha sido Pierre Bayle: «su sistema [el aristotélico] (...) es incapaz de dar pruebas de la inmortalidad de nuestra alma, pero muy capaz de dar pruebas de que nuestra alma es mortal» (P. Bayle, «Pomponazzi», en Diccionario histórico y crítico. Selección. trad. de J. Bayod, Barcelona, Círculo de Léctores, 1996, p. 271). 
orientarse a fundamentar las verdades de la fe, ni tampoco debe estar limitada por ellas ${ }^{46}$. Frente a la interpretación pro-radical de intérpretes como Pine, para Perrone, el hecho de que Pomponazzi establezca en el capítulo XIV del De immortalitate una explicación de la religión desde el ámbito de la razón (esto es, como una forma de control social del vulgo, un instrumentum regni que tiene que ver con el valor moral y político de la religión ${ }^{47}$, pero no necesariamente con el valor de la fides, al menos, de la Christiana fides), no hace sino confirmar el carácter irreprochable de la postura de Pomponazzi: de hecho, incluso aunque éste no sea sincero, el problema de su «sinceridad» resulta al cabo un problema «neutro»y, por otro lado, «irrelevante» ${ }^{48}$.

Es cierto que la cuestión sobre la fe o descreencia de Pomponazzi es un terreno impracticable, en el que no se puede entrar sin hacer juicios de intenciones, carentes de fundamento textual. Pero no podemos estar de acuerdo en que la sinceridad de Pomponazzi sea un problema irrelevante, y sobre todo que se agote en su neutralidad. Un problema, en sentido aristotélico, se plantea sólo si tiene algún interés y normalmente permite determinar hacia qué lugar de los polos opuestos se orienta la verdad.

No es éste el lugar para abordar el amplio y complejo debate sobre el pretendido discurso entre líneas que plantea el pensamiento de Pomponazzi. Me limitaré por tanto a defender modestamente que la problemática aquí abordada parece dar la razón a la interpretación clásica, aquella que considera que las palinodias y retractaciones de Pomponazzi actúan esencialmente como fórmulas ad cautelam de un postura poco piadosa o, en todo caso, crítica respecto no sólo de las leges (como propone Perrone), sino también del valor epistémico de la fe $\mathrm{fe}^{49}$.

En una versión reciente de esta línea interpretativa, Sergio Landucci nos ha recordado cómo es imposible cerrar los ojos ante las dislocaciones polé-

46 Ibid., p. XCI.

47 P. Pomponazzi, Tratado sobre la inmortalidad del alma, XIV, 140-42.

48 Ibid., pp. XCV-XCVI. En la conclusión de un reciente artículo dedicado al determinismo naturalista del De incantationibus, la experta en Pomponazzi parece insinuar una total ausencia de religiosidad por parte del filósofo, (V. Perrone, «La teologia di Pomponazzi: Dio e gli dei», en Pietro Pomponazzi. Tradizione e dissenso, ed. de M. Sgarbi, Florecia, Olschki, 2010, p 129).

${ }^{49}$ M. Pine, P. Pomponazzi radical Philosopher of the Renaissance, 1986, p. 123; G. Di Napoli, L'immortalità dell'anima nel Rinascimento, Turín, SEI, 1963, p. 265; M. Á. Granada, Cosmología, religión y politica en el Renacimiento: Ficino, Savonarola, Pomponazzi, Maquiavelo, Barcelona, Anthropos, 1988, pp. 121-134 y 196-217. 
micas y poco «piadosas» que salen a la luz en cada tratado de Pomponazzi ${ }^{50}$. Para empezar, el estudioso italiano nos recuerda que el carácter «neutro» del problema de la inmortalidad había sido negado por un adversario bien conocido de Pomponazzi en un texto de 1503. En efecto, Agostino Nifo, en su De intellectu, se había ya mostrado contrario a todos aquellos que sostenían que la inmortalidad era un problema neutrum. De acuerdo con Nifo, la inmortalidad, lejos de ser un problema indeciso, debía afirmarse no sólo por la autoridad de la fe cristiana, «sino por las razones y demostraciones a priori y a posteriori» que podían ofrecerse de ella ${ }^{51}$.

Hemos visto que Pomponazzi no se plantea la eternidad del mundo como un problema en términos aristotélicos, sino más bien como una proposición. Es más, se trata de una proposición altamente verosímil, en la medida en que por vías distintas (a partir de la definición del movimiento y de concepción aristotélica de la naturaleza divina) las alternativas de creación ex nibilo o de novo se presentan como sencillamente ininteligibles, esto es, han sido relativamente refutadas. Por lo demás, la apelación al carácter neutral de ciertos problemas filosóficos no implica en absoluto una puerta abierta al escepticismo o al fideísmo. La neutralidad no es absoluta, sino relativa a la verdad apodíctica. Un problema neutral es un problema potencialmente a la espera de una argumentación que lo incline en una dirección verosímil, dialéctica. En la tradición aristotélica, el saber filosófico contiene en realidad pocas verdades con carácter demostrativo, necesitando en muchas ocasiones la dialéctica ${ }^{52}$. Los instrumentos de la dialéctica y en concreto, el método diaporético sirve justamente para resolver la dirección de un problema que se debate entre proposiciones dilemáticas y del que no cabe deducción apodíctica, sino sólo la verosimilitud derivada, cuando es ello posible, de la refutación (élenchos) de uno de los polos del problema. Que la dialéctica permita una discriminación entre lo verdadero y lo falso, lo dice Aristóteles expresamente ${ }^{53}$. Y justamente, como ha señalado recientemente Zanatta, y tan-

50 S. Landucci, La doppia verità. Conflitti di ragione e fede tra Medioevo e prima modernità, Milán, Feltrinelli, 2006, pp. 108 y sigg.

51 A. Nifo, De intellectu, ed. de L. Spruit, Leiden, Brill, 2011, I, x, p. 158. La misma idea se reitera en el cap. LXXXIV del L'immortalità dell'anima, p. 430. Pomponazzi en el Defensorium insistirá en el carácter neutro de la eternidad del mundo, y en la independencia de esta neutralidad (que se refiere sólo a los razonamientos apodícticos) respecto de las verdades de la fe y la autoridad católica (cfr. P. Pomponazzi, Defensorium, Bolonia, Iustinianum de Rubiera, 1519, cap. XXXV).

52 Aristóteles, Top., I, 1, 101a

53 Ibid., VIII, 14, 163b10-14. 
tos otros anteriormente, el procedimiento dialéctico es usado abundantemente por Aristóteles en el ámbito de la filosofía natural, partiendo de la crítica de las alternativas enunciadas por otros pensadores ${ }^{54}$.

Es obvio, por lo demás, que aunque Pomponazzi considerara la dialéctica como una forma de conocimiento decididamente distinto de la demostración apodíctica, en ningún momento la ha equiparado a la sofística o a una retórica vacía. Desde este punto de vista, Pomponazzi no puede ser caracterizado como un agnóstico o un escéptico en sentido estricto. Las proposiciones dialécticas no son en Aristóteles, ni lo serán en Pomponazzi, una forma de ignorancia, sino una forma no demostrativa de conocimiento. Y es éste el horizonte epistémico en el que se mueven de las tres grandes obras de Pomponazzi: tratar de recorrer dialécticamente el problema de la inmortalidad, del destino y de los fenómenos pretendidamente sobrenaturales, permitiendo abrir un ámbito de verosimilitud a través de la refutación de las diferentes alternativas, tomando como punto de partida tanto la exegesis de Aristóteles como las premisas de la razón natural.

Si retomamos ahora la tesis de Perrone, ésta se cifraba en considerar que aunque Pomponazzi pudiera pensar que la mortalidad del alma o las hipótesis deterministas resultaban más probables que los contenidos de la fe cristiana, y que el mundo eterno basado en el pensamiento de Aristóteles parecía más verosímil que el mundo creado ex nihilo, ex novo o de novo, esto, siempre según Perrone, no afectaría en absoluto a la fe cristiana, pues al moverse en otro plano podía perfectamente permanecer intacta.

Ahora bien, yo no concedería esta mutua independencia con tanta facilidad. Pues la fe es siempre fe en algo, esto es, en un conjunto de contenidos.

${ }^{54}$ M. Zanatta, «Dialéctica y ciencia en Aristóteles», Anuario Filosófico, 35, 2002, pp. 44 y 50-51. La cuestión está sujeta a controversia incluso hoy, pues, ciertamente, las pretensiones epistémicas de la dialéctica fueron puestas en entredicho por el propio Aristóteles, al condenarla (en Metaph, IV, 2, 1004b25) a ser una tentativa o crítica (peirastiké) de aquello mismo de lo que la filosofía es ciencia (gnoristiké). Sobre la importancia de la dialéctica y el carácter diaporético de la física aristotélica, aparte del texto de Zanatta, véase G. R. de Echandía, «Introducción», en Aristóteles, Física, Gredos, 1995, pp. 46-48. En cualquier caso, es obvio que al menos Pomponazzi tiene en mente más bien la interpretación "positiva» de la dialéctica (no sólo útil, sino necesaria para la filosofía) común al aristotelismo renacentista (y a la tradición aristotélica, tanto antigua como medieval) que puede ejemplificarse mediante el caso de Nifo (que compuso varias obras abordando la cuestión), como muestra un reciente y espléndido estudio sobre la materia (cfr. M. Spranzi, The Art of Dialectic between Dialogue and Rhetoric: The Aristotelian Tradition, Amsterdam, John Benjamins, 2011, pp. 99-132). 
La fe además es el fundamento de la religión, por lo que tratar de disociar la fe genérica de los contenidos de la religión, en este caso de la religión cristiana, parece al menos aquí problemático. Y el problema es justamente que la fe y la razón no son compartimentos estancos, sino que tienden a comunicarse a través de una serie de contenidos. Su actividad se entrecruza al dirigirse hacia objetos comunes: una cosmología, una visión moral del hombre, una antropología. Para el caso que nos ocupa, este encuentro no puede ser pacífico, en cuanto que ambas desarrollan un discurso divergente y que a la vez se pretende verdadero sobre cuestiones teóricas y prácticas. Tratar de no ver el conflicto es cerrar los ojos. Y es el propio Pomponazzi el que a veces no puede evitar confrontar directamente los dos planos y tachar, por ejemplo de «delirios que repugnan a la filosofía», de postulado «arbitrario», de ideas «irracionales», las tesis que afirman que el alma sea una sustancia espiritual e inmortal al mismo tiempo que la forma del cuerpo ${ }^{55}$. Esto es, básicamente, las tesis del credo cristiano y tomista.

No sólo hay vasos comunicantes entre fe y filosofía. Sucede además, que la fe y la religión, son a su vez objeto de la filosofía, que se permite dar una explicación de sus fundamentos (los milagros, las profecías) e incluso de su razón de ser, que es, como hemos visto, de índole práctica y moral. No hay nada que permita pensar que Pomponazzi asume dos formas de conocimiento con contenidos contradictorios y al mismo tiempo autónomos e independientes (donde hay contradicción no puede haber independencia). Sí parece coherente, en cambio, pensar que Pomponazzi concibe estas dos formas de conocimiento no en un mismo sujeto cognoscitivo, sino en el reparto epistemológico interno del cuerpo social. En éste, como sabemos por el De immortalitate animae, la mayor parte de los hombres (sea por su naturaleza, sea por falta de tiempo, sea por las exigencias de la división del trabajo) no puede dedicarse a la filosofía ni a la indagación racional. En este sentido, la mayoría de los hombres, como hemos visto, debe guiarse por las ideas propuestas por la prudencia del legislador y por las «fábulas» de la tradición. Otra parte de la sociedad, puede permitirse sin embargo la búsqueda del conocimiento racional, de la verdad, o al menos de las hipótesis menos contradictorias o más probables. Es entre estas dos partes de la sociedad en donde es posible la incomunicación y la perfecta autonomía entre fe y razón, siempre que toda ofensa o escándalo no se haga público y que toda impiedad o resultado poco edificante venga protegido con una forma de cobertura justamente inatacable e irreprochable.

55 P. Pomponazzi, Tratado sobre la inmortalidad del alma, IX, pp. 73-74. 
No parece entonces demasiado suspicaz pretender que Pomponazzi utiliza las retractaciones como baluartes defensivos y formulas ad cautelam. Landucci, recordando aquí la vieja fórmula de Strauss, señala que un criterio para establecer si un autor utiliza tácticas de escritura defensivas es comprobar si éste se las atribuye a otro ${ }^{56}$. Pomponazzi en este sentido no se limita a atribuírselas a otros (Platón, Aristóteles, Averroes), sino que también parece complacerse en ellas. Y no es impensable que creyera, como Galeno en el texto citado por él, que era preferible aceptar públicamente lo que pensara la «canalla» antes que ser lapidado. Máxime porque no se trataba sólo de una cautela cuyo fin fuera su seguridad personal. Sus comentarios sobre las relaciones entre la filosofía y la teología-ley-costumbre muestran que no hay ninguna pretensión de que la filosofía pueda suplantar o presentarse como alternativa a la religión en el ámbito social. Por todo ello, bien puede aceptarse que la sumisión de Pomponazzi a la autoridad de la Iglesia no tiene nada de insincero. Al fin y al cabo éste se muestra convencido de que algunas verdades no están hechas para el vulgo ni para ciertos teólogos:

Ciertamente esto [la explicación naturalista de los fenómenos sobrenaturales] no debe ser transmitido al vulgo, pues éste no es apto para tales arcanos y no cree sino lo que ve o lo que está acostumbrado a ver. Cuidado también con los sacerdotes inexpertos que sermonean sobre estas cosas. Pues he aquí muchas veces la causa patente de que los filósofos hayan sido expulsados de las ciudades, o encarcelados, o lapidados y sometidos al último castigo ${ }^{57}$.

\section{Bibliografía}

Aristóteles, Tópicos (1982). Ed. de M. Candel, Madrid, Gredos.

- Física (1995). Ed. de Guillermo R. de Echandía, Madrid, Gredos.

Bayle, Pierre (1996). «Pomponazzi», en Diccionario histórico y crítico. Selección. trad. de J. Bayod, Barcelona, Círculo de Lectores, pp. 253-288.

Bianchi, Luca (2008). Pour una historie de la «double vérité», París, Vrin.

Dales, Richard C. (1990). Medieval Discussions on the Eternity of the World, Nueva York, Brill, 1990.

Di Napoli, Giovanni (1963). L'immortalità dell'anima nel Rinascimento, Turín, SEI.

56 S. Landucci, La doppia verità, p. 114.

57 P. Pomponazzi, De incantiationibus, XII, p. 131. 
Falconi, Carlo (1987). Leone X: Giovanni de Medici, Milán, Rusconi.

Granada, Miguel Ángel (1988). Cosmología, religión y politica en el Renacimiento: Ficino, Savonarola, Pomponazzi, Maquiavelo, Barcelona, Anthropos.

Guicciardini, Francesco (1971). Storia d'Italia, ed. de S. Seidel Mechi, Turín, Einaudi.

Kovach, Francis J. (1974). «The Question of the Eternity of the World in St. Bonaventure and St. Thomas», Southwestern Journal of Philosophy, 5/2, pp. 141-172.

Landucci, Sergio (2006). La doppia verità. Conflitti di ragione e fede tra Medioevo e prima modernità, Milán, Feltrinelli.

Michon, Cyrille (2004). Thomas d'Aquin et la controverse sur "L 'éternité du monde». Traités sur "L' Éternité du monde» de Bonaventure, Thomas d'Aquin, Peckham, Boèce de Dacie, Henri de Gand et Guillaume d'Ockham, París, Flammarion.

Nifo, Agostino (2011). De intellectu, ed. de L. Spruit, Leiden, Brill.

- L'immortalità dell'anima (2009). Ed. de J. M. García Valverde, Milán, Aragno.

Perrone, Vittoria (2010). «La teologia di Pomponazzi: Dio e gli dei», en Pietro Pomponazzi. Tradizione e dissenso, ed. de M. Sgarbi, Florencia, Olschki, pp. 107-129.

Poppi, Antonino (2010). «Consenso e dissenso del Pomponazzi con il subtilissimus et religiosissimus Ioannes Scotus», en Pietro Pomponazzi. Tradizione e dissenso, ed. de M. Sgarbi, Florencia, Olschki, pp. 3-39.

Pomponazzi, Pietro (1966-1970). Corsi inediti dell'insegnamento padovano. Super libello de substantia orbis expositio et quaestiones quattor, 2 vols., ed. de A. Poppi, Antenore, Padova.

- (1965). In proemium Commentatoris III physicae auscultationis, en B. Nardi, Studi su Pietro Pomponazzi, Florencia, Le Monnier.

- (2011). Quaestio de genitis ex putri materia, en PERRONE, Vittoria, "La stagione delli frumenti. Due lezioni di Pomponazzi sulla generazione spontanea», Bruniana \& Campanelliana, 17/1, pp. 199-222.

- (2010). Tratado sobre la inmortalidad del alma, ed. y trad. de J. M. García Valverde, Madrid, Tecnos.

- (1999). Trattato sull'immortalità dell'anima, ed. de V. Perrone, Florencia, Olschki.

- (1957). Libri Quinque de Fato, de Libero Arbitrio et de Praedestinatione, ed. de R. Lemay, Lugano.

- (2011). De incantationibus, ed. de V. Perroni, Florencia, Olschki.

- (2011). Apologia, ed. de V. Perrone, Florencia Olschki.

- (1519). Defensorium, Bolonia, Iustinianum de Rubiera. 
Pastor, Ludwig (1910). Historia de los papas, ed. de R. P. Ramón Ruiz, vol. VIII, Barcelona, Gustavo Gili.

Pine, Martin (1986). P. Pomponazzi Radical Philosopher of the Renaissance, Padua, Antenore.

Sacrorum conciliorum nova et amplissima collectio, 53 vols., ed. de G. D. Mansi, París, H. Welter, 1901-1927.

Spranzi, Marta (2011). The Art of Dialectic between Dialogue and Rhetoric: The Aristotelian Tradition, Amsterdam, John Benjamins.

Zanatta, Marcello (2002). «Dialéctica y ciencia en Aristóteles», Anuario Filosófico, 35, pp. 25-52.

Recibido: 10/12/2012

Aceptado: 7/02/2013 